\title{
Pattern formation in reaction-diffusion systems with piecewise kinetic modulation: An example study of heterogeneous kinetics
}

\author{
Michal Kozák $\odot,{ }^{1}$ Eamonn A. Gaffney, ${ }^{2}$ and Václav Klika ${ }^{1, *}$ \\ ${ }^{1}$ Department of Mathematics, FNSPE, Czech Technical University in Prague, Prague, Czech Republic \\ ${ }^{2}$ Wolfson Centre for Mathematical Biology, Mathematical Institute, University of Oxford, United Kingdom
}

(Received 27 August 2019; published 31 October 2019)

\begin{abstract}
The study of pattern emergence together with exploration of the exemplar Turing model is enjoying a renaissance both from theoretical and experimental perspective. Here, we implement a stability analysis of spatially dependent reaction kinetics by exploring the effect of a jump discontinuity within piecewise constant kinetic parameters, using various methods to identify and confirm the diffusion-driven instability conditions. Essentially, the presence of stability or instability in Turing models is a local property for piecewise constant kinetic parameters and, as such, may be analyzed locally. In particular, a local assessment of whether parameters are within the Turing space provides a strong indication that for a large enough region with these parameters, an instability can be induced.
\end{abstract}

DOI: 10.1103/PhysRevE.100.042220

\section{INTRODUCTION}

Understanding pattern formation is one of the major issues not only in developmental biology but across many different disciplines. A seminal mechanism for self-organization emergence was proposed by Alan Turing in 1952 [1]. He considered two biochemicals (the so-called morphogens) which diffuse and interact with each other via reaction terms and demonstrated that a small fluctuation of their concentrations around a steady state can be heterogeneously amplified for suitable reaction kinetics coupled with diffusion. Therefore spatially nonhomogeneous steady states, that is, patterns can emerge. Mathematically, this symmetry breaking mechanism was described as a diffusion-driven instability (DDI) of the steady state in a reaction-diffusion system (RD system). Comparing theoretical predictions and real patterns seen in nature, it has been reported that Turing models may indeed feature in, for example, the formation of wild cat skin patterns [2], tumor vascularization [3], hair follicle localization [4,5], pigmentation of zebrafish [6], rugae formation [7], and digit patterning [8].

For many decades, one of the biggest issues for the plausibility of a Turing system in biological development was the lack of identification of morphogens and confirming the molecular details match those required for patterning, though recent studies show extensive promise [9-11]. Furthermore, there is in addition the suggestion that the combination of Wolpert's positional information hypothesis $[12,13]$ with Turing's mechanism may increase the applicability of both $[14,15]$.

Many Turing systems have been proposed and analyzed, both analytically and using numerical experiments. The majority of these studies consider only two species. The exemplar models are the Gierer-Meinhardt model [16] describing the growth of hydra; the Schnakenberg model [17] describing

\footnotetext{
*vaclav.klika@fjfi.cvut.cz
}

a chemical reaction exhibiting limit-cycle behavior or the Thomas model [18] describing chemical reaction of oxygen and uric acid in the presence of the enzyme uricase; see [19] for a review of these models. A standard question is to find the Turing space, that is the set of parameters for which Turing's diffusion-driven instability occurs, whereby the system's steady state is stable to homogeneous perturbations but unstable to heterogeneous perturbations assuming the domain size is sufficiently large to support the growth of the unstable perturbations.

The resulting patterns of the Turing model with constant coefficients are typically highly periodic and hence fail to capture key features of many real systems, where the patterns significantly change in space. The reason is intuitive-the model is too simplistic. The addition of spatial dependency in model parameters is a natural way to extend the Turing model and probably an intuitive way of potentially generating wavelength variation [20]. The Gierer-Meinhardt model [16], for example, is originally based on a source with a gradient; even Turing in his paper [1] discussed that "Most of an organism, most of the time, is developing from one pattern into another, rather than from homogeneity into a pattern," suggesting significant spatial dependency may be initially present before the impact of the Turing instability.

There are many examples of spatial irregularity in patterns where wavelength variation is manifest, for example, a distribution of mouse or cat whiskers [21], alternating thin and thick stripes of Lionfish [22], and emergence of fingers [15]. In these cases heterogeneous pattern modulation is a crucial feature of the self-organization and, as a result, we would like to capture this phenomena in a modeling framework for the emergence of self-organization. Similarly, the heterogeneity of the environment in landscape ecology [23] or the influence of geometric confinement of human embryonic stem cells [24] illustrate important and heterogeneous aspects of patterning systems. Such ubiquitous examples emphasize the critical importance of studying the impact of heterogeneity and its effects on the properties of self-organizing systems. 
(a)

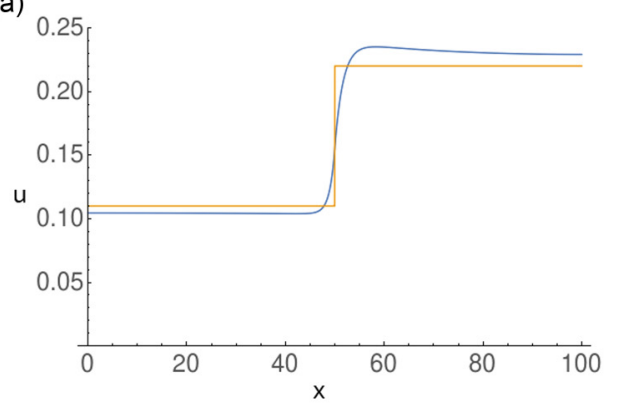

(c)

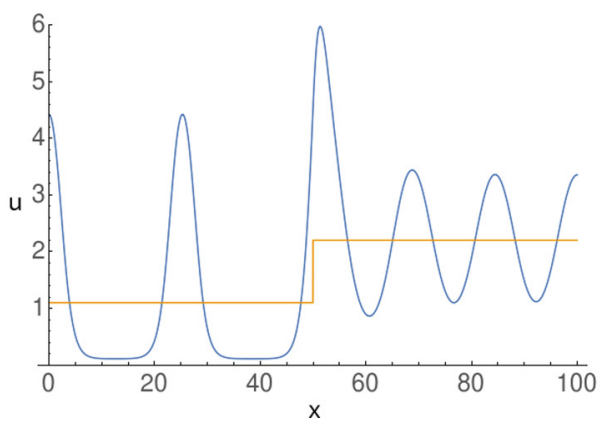

(b)

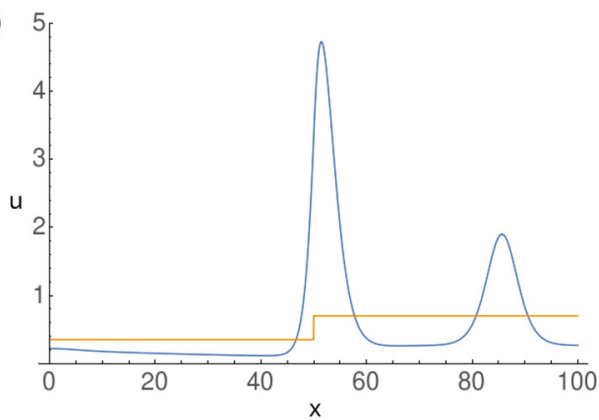

(d)

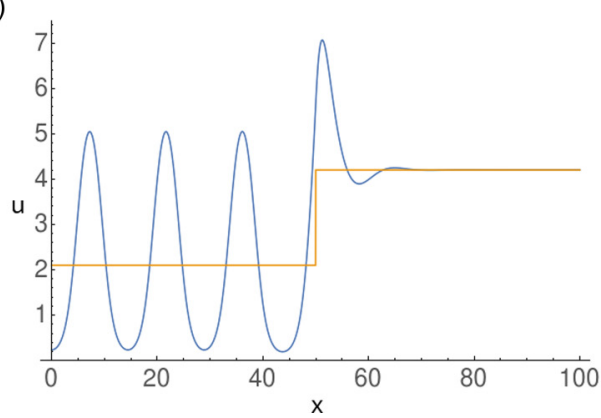

FIG. 1. Plot of the long-time (close to steady state) activator concentration $u(x)$ from simulations of the system (1) with Schnakenberg kinetics (4) on the domain [0,100] with zero-flux boundary conditions, and parameters as follows: $d_{1}=1, d_{2}=100, s=0.5, a=0.1$, and (a) $b=0.01$, (b) $b=0.25$ (both outside the Turing space for $s=0$ ), (c) $b=1$, (d) $b=2$ (both inside the Turing space for $s=0$ ). These have been solved by MATHEMATICA (for more details see below). Note the different vertical scales in the above plots.

However, the standard procedure of stability analysis is not easily extendable to the case with spatially dependent coefficients as, for example, a homogeneous steady state does not exist in general given spatially dependent kinetics. Probably the most well-understood effect of heterogeneity comes from the shadow limit in Gierer-Meinhardt kinetics using spike solutions [25-27] but note that this requires the applicability of the infinite or arbitrarily large diffusion coefficient in one of the species. Further the case of a spatially dependent diffusion coefficient was analyzed in [28] with a step function representing the dependency. Heterogeneity in the reaction kinetics was analyzed numerically [22] and limited analytical progress in stability analysis has been established with spatial dependency in the kinetics. Examples include asymptotically small, spatially dependent, linear gradients of morphogen source [29-31], a cosine spatial dependence in a coefficient of the kinetics [32] and a step function, independent of morphogen concentration, added to the kinetics [33]. Finally, very recently a rather general stability analysis with spatial heterogeneity in reaction kinetics was successfully proposed including a transition from one pattern into another [34]. Essentially the only situation this approach using WKBJ asymptotics does not cover is when the spatial oscillations are rapid, which is a fundamental feature induced by the jump discontinuity in the kinetics that we consider here.

This article deals with a system with a spatially dependent coefficient in the linear term of the activator kinetics which introduces very different challenges to the above described cases. For analytical convenience, we assume a onedimensional space. We consider the following RD system:

$$
\begin{array}{ll}
\partial_{t} u=d_{1} \partial_{x x} u+f_{1}(u, v)+h(x) u, & x \in(0, L), \\
\partial_{t} v=d_{2} \partial_{x x} v+f_{2}(u, v), & x \in(0, L),
\end{array}
$$

with Neumann boundary conditions,

$$
\begin{aligned}
& \frac{\partial u}{\partial n}=0, \quad \text { at } x=0, L, \\
& \frac{\partial v}{\partial n}=0, \quad \text { at } x=0, L,
\end{aligned}
$$

where $h(x)$ is a step function defined as

$$
h(x)= \begin{cases}0 & x \in[0, \xi), \\ s & x \in[\xi, L] .\end{cases}
$$

First for reasons that shall become evident, we need to assess what will be denoted as a pattern since the standard definition is not sufficient because inhomogeneity is always present due to the forced jump $s$ at location $\xi$. We illustrate the effect of the step function via numerical simulations, considering system (1) with Schnakenberg kinetics,

$$
\begin{aligned}
& f(u, v)=a-u+u^{2} v, \\
& g(u, v)=b-u^{2} v,
\end{aligned}
$$

where $a, b$ are positive parameters. Let the step, with a size $s=0.5$, be located in the middle of the domain $\xi=L / 2$ and consider the remaining parameters $d_{1}, d_{2}, a, b$ to be outside ( $b \in\{0.01,0.25\})$ or inside $(b \in\{1,2\})$ the Turing space for $s=0$.

As we can see in Fig. 1 (blue wavy line), a spatial inhomogeneity occurs in each of the solutions. Note (i) the different amplitudes of the pattern in the two parts of the domain (as already observed, e.g., in [33]) and (ii) the different periods [see Fig. 1(c)]. Characterizing such patterning behavior in Fig 1(c), within the context of emergent Turing self-organization from a linearized system with spatial heterogeneity in the form of 

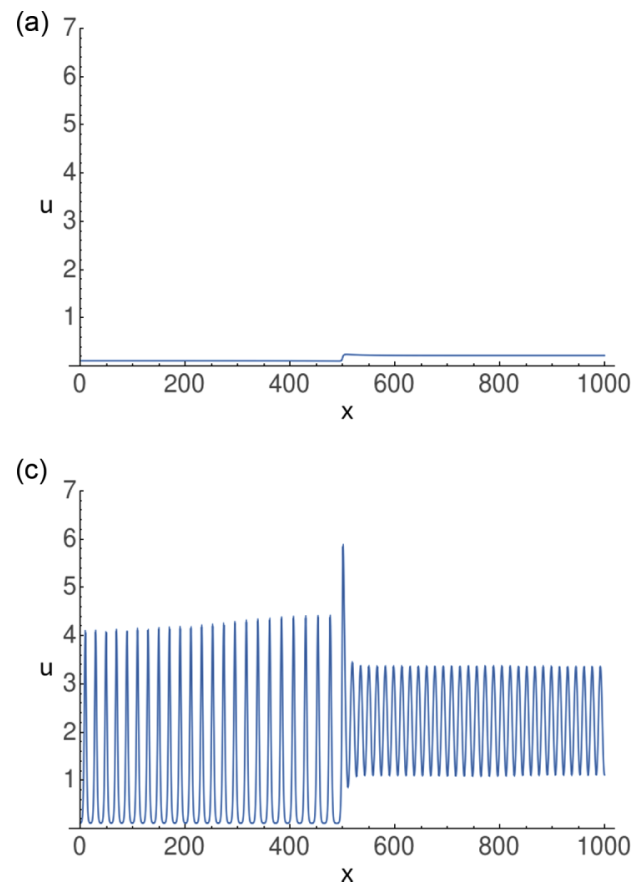

(b)

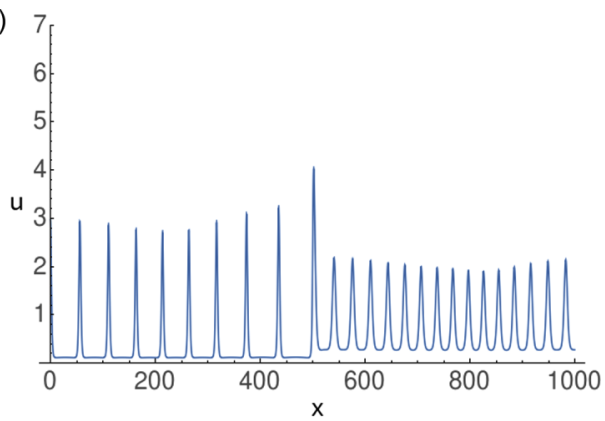

(d)

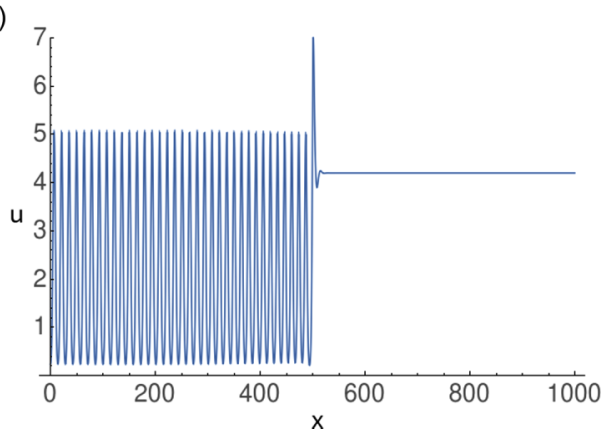

FIG. 2. Plot of the long-time (close to steady state) activator concentration $u(x)$ from simulations of the system (1) with the same values as in Fig. 1 apart from $L=1000$. The vertical axis is chosen equal for all plots. This figure illustrates the effect of larger $L$ which is key for discussion of what a "pattern" should mean in systems with heterogeneous kinetics.

a step function premultiplying a linear term, constitutes the overarching aim of this study.

As mentioned above, not all the inhomogenous solutions displayed in Fig. 1 correspond to a pattern, however. To represent genuine self-organization, rather than being passively slave to the step function, such stationary solutions should have spatial oscillations extending to the domain edge on at least one side of the step even as the domain size is increased, for sufficiently large domain sizes. This requirement follows from an observation that a Turing pattern is characterized by a finite number of frequencies that appear in the pattern. Thus an increase in the domain size should result in pattern repetition over the whole domain once a critical domain size is surpassed. Therefore we plot stationary solutions to the system with the same parameters except a larger domain size, $L=1000$, in Fig. 2 . By comparison, we can deduce that Fig. 2(a) is not a pattern as the inhomogeneity is localized only around the point of the step $\xi$ while being of the order of the step $s$. Hence we disregard such cases in the context of pattern. On the contrary, in the other examples the inhomogeneity perseveres on the whole domain; thus such cases are denoted as a pattern.

Hence our detailed objectives are as follows: (i) to determine if a pattern emerges or not; (ii) to undertake a more specific pattern classification examining the parameter spaces for when the system will exhibit each prototype of a stationary solution represented by the plots in Figs. 1(a) no pattern, 1(b) right-sided pattern, 1(c) global pattern, and 1(d) left-sided pattern $^{1}$.

\footnotetext{
${ }^{1}$ Note the discrepancy between plots in Figs. 1(b) and 2(b): The former case is denoted as a right-sided pattern whereas the latter
}

Let us further make the following interesting observation. We consider step functions $\bar{u}(x), \bar{v}(x)$ defined as

$$
\bar{u}(x)=\left\{\begin{array}{ll}
\bar{u}^{L} & x \in(0, \xi) \\
\bar{u}^{R} & x \in(\xi, L)
\end{array}, \quad \bar{v}(x)= \begin{cases}\bar{v}^{L} & x \in(0, \xi) \\
\bar{v}^{R} & x \in(\xi, L)\end{cases}\right.
$$

where

$$
\begin{aligned}
f\left(\bar{u}^{L}, \bar{v}^{L}\right) & =0=g\left(\bar{u}^{L}, \bar{v}^{L}\right), \\
f\left(\bar{u}^{R}, \bar{v}^{R}\right)+s \bar{u}^{R} & =0=g\left(\bar{u}^{R}, \bar{v}^{R}\right) .
\end{aligned}
$$

Note that in Turing-like problems the existence of a homogeneous steady state is assumed, guaranteeing existence of the step functions (5). The long-time solution for the variable $u$ seems to be either approaching $\bar{u}(x)$ in the case without pattern, or oscillating around $\bar{u}(x)$ in the case of pattern. This leads us to a hypothesis that the behavior of the long-time solution could be deduced from attracting properties of the system around $\bar{u}(x)$ and therefore the system characterizations could be similar to the conditions of diffusion driven instability evaluated for the system (with a constant coefficient) considered separately on intervals $(0, \xi)$ and $(\xi, L)$. In particular, such prospects will be considered more precisely and systematically below.

patterns globally. The only change is in the value of parameter $L$ and it seems, roughly speaking, $L$ should be large enough and cases where the pattern cannot form solely because of the domain are generally classified as patterning systems nonetheless. Please note that this is analogous to the classical Turing approach where a pattern appears only once a certain minimal domain size is reached so that the first eigenmode fits within the domain and can be excited. 
In Sec. II A, we consider system (1) with affine kinetics $f, g$ and linearize about the steady state. The system behaves similarly to the original one, but where the propensity to pattern is replaced by the existence of an unbounded solution as time tends to infinity. Hence, we analyze the growth or decay of perturbations in the linearized system, with the implicit assumption that the eventual steady state of the full system will inherit the same spatial frequency of heterogeneity of one of the unstable, diffusively driven, growing solutions exhibited by the linearized system. Such implicit assumptions are standard in linear stability analysis [19]. Furthermore when the linear system exhibits no growing solutions, even for an arbitrarily large domain, the system does not exhibit a Turing instability. Thus, in summary, we analytically solve the stationary problem and proceed to analyze the linear stability of the steady state via a spectral approach with the results compared to extensive numerical simulations of the full evolution problem.

We then complement this spectral approach by a boundary layer analysis which further suggests that conditions for the one-sided pattern are related to Turing conditions considered separately on each subinterval. In addition, this boundary layer analysis indicates that spatial frequency of the emerging pattern is determined independently in the two subintervals while the boundary layer highlights how the patterns match in the interval around the step at $x=\xi$ of the step functions. Finally, the hypothesized local conditions for a Turing instability are verified by a sweep of numerical solutions to the evolution problem with Schnakenberg kinetics before drawing conclusions in Sec. IV.

\section{ANALYTICAL APPROACH}

\section{A. Spectral theory}

Let us consider system (1) with general kinetics. The behavior of such a system is usually inferred from the behavior of the linearized system, which describes the evolution around the steady state where the influence of linear kinetics exceeds the influence of nonlinear parts of kinetics. Hence characterizations via linear systems are generally true at least for small enough initial perturbations of the steady state.

However, as mentioned in Sec. I, since $s$ is nonzero, we cannot expect the steady state to be homogeneous. Note that resolving the steady state requires solving a system of nonlinear elliptic partial differential equations. Further, if we consider an expansion of the nonlinear kinetics around this nonhomogeneous steady state, the resulting coefficients in the linear terms would be strongly spatially dependent which may prevent analytical tractability. Hence we focus on the case of linear kinetics where the step occurs only in a single linear kinetic term. Then, generalization to linear kinetics with jumps in every kinetic term is implemented.

Consider the following RD system with affine kinetics and the step function $h(x)$ defined above (3),

$$
\begin{aligned}
& \partial_{t} u=d_{1} \partial_{x x} u+b_{10}+\left(b_{11}+h(x)\right) u+b_{12} v \\
& \partial_{t} v=d_{2} \partial_{x x} v+b_{20}+b_{21} u+b_{22} v,
\end{aligned} \quad x \in(0, L),
$$

with Neumann boundary conditions (2).
The advantage of considering affine kinetics, even though they are not standard, is that the system then in general describes the dynamics not only around a trivial solution, but also around a nontrivial one, as will be apparent below and in particular in Appendix A 1.

\section{Analytic solution to the stationary problem}

Our first task is to find a stationary solution to the system (6) explicitly. The following approach is not novel, moreover the complexity and length of the steady-state solution is out of proportion to the usefulness of explicitly stating it and hence we highlight the important features only.

Since $\bar{u}(x), \bar{v}(x)$ defined in (5) and $h(x)$ are step functions at $\xi$, it is easier to consider the system (6) restricted to the interval $(0, \xi)$ and $(\xi, L)$ separately and to use appropriate connecting conditions at $\xi$. From now on the upper index $L$ corresponds to the system on $(0, \xi)$, while the index $R$ corresponds to the interval $(\xi, L)$, respectively. For the perturbations,

$$
\begin{array}{ll}
\tilde{u}^{L}=u^{L}-\bar{u}^{L}, & \tilde{v}^{L}=v^{L}-\bar{v}^{L}, \\
\tilde{u}^{R}=u^{R}-\bar{u}^{R}, & \tilde{v}^{R}=v^{R}-\bar{v}^{R},
\end{array}
$$

we obtain an equivalent steady system of the form,

$$
\begin{array}{ll}
0=d_{1} \partial_{x x} \tilde{u}^{L}+b_{11} \tilde{u}^{L}+b_{12} \tilde{v}^{L}, & \\
0=d_{2} \partial_{x x} \tilde{v}^{L}+b_{21} \tilde{u}^{L}+b_{22} \tilde{v}^{L}, & \text { in }(0, \xi), \\
0=d_{1} \partial_{x x} \tilde{u}^{R}+\left(b_{11}+s\right) \tilde{u}^{R}+b_{12} \tilde{v}^{R}, & \\
0=d_{2} \partial_{x x} \tilde{v}^{R}+b_{21} \tilde{u}^{R}+b_{22} \tilde{v}^{R}, & \text { in }(\xi, L),
\end{array}
$$

with boundary and connecting conditions $\left[C^{1}(0, L)\right.$ solutions of the form $u=u^{L} h(\xi-x)+u^{R} h(x-\xi), v=v^{L} h(\xi-x)+$ $\left.v^{R} h(x-\xi)\right]$,

$$
\begin{aligned}
\frac{\partial \tilde{u}^{L}}{\partial x}(0) & =\frac{\partial \tilde{v}^{L}}{\partial x}(0)=0, \quad \frac{\partial \tilde{u}^{R}}{\partial x}(L)=\frac{\partial \tilde{v}^{R}}{\partial x}(L)=0, \\
\frac{\partial \tilde{u}^{L}}{\partial x}(\xi) & =\frac{\partial \tilde{u}^{R}}{\partial x}(\xi), \quad \frac{\partial \tilde{v}^{L}}{\partial x}(\xi)=\frac{\partial \tilde{v}^{R}}{\partial x}(\xi), \\
\tilde{u}^{R}(\xi)-\tilde{u}^{L}(\xi) & =\bar{u}^{L}-\bar{u}^{R}, \quad \tilde{v}^{R}(\xi)-\tilde{v}^{L}(\xi)=\bar{v}^{L}-\bar{v}^{R} .
\end{aligned}
$$

Tildes will be omitted henceforth.

The simple way to solve such systems is to transform the matrix $\mathbf{B}=\left(\begin{array}{ll}b_{11} & b_{12} \\ b_{21} & b_{22}\end{array}\right)$ into its Jordan normal form on both subsystems separately, considering the boundary conditions but not the connecting ones, and completing the calculation of the steady state by employing the connecting conditions, as implemented in [33].

An interesting issue is that the resulting form of the solution to each subsystem depends on the sign of $\left(\operatorname{tr}^{2} \mathbf{A}^{L, R}-\right.$ $\left.4 \operatorname{det} \mathbf{A}^{L, R}\right)$, where $\mathbf{A}^{L, R}$ denote matrices of coefficients readily written in terms of normalized linear terms for both subsystems, i.e.,

$$
\mathbf{A}^{L}=\left(\begin{array}{ll}
b_{11} / d_{1} & b_{12} / d_{1} \\
b_{21} / d_{2} & b_{22} / d_{2}
\end{array}\right), \mathbf{A}^{R}=\left(\begin{array}{cc}
\left(b_{11}+s\right) / d_{1} & b_{12} / d_{1} \\
b_{21} / d_{2} & b_{22} / d_{2}
\end{array}\right) .
$$


Particularly, the signs of the following two terms are important:

$$
\begin{array}{r}
\left(d_{2} b_{11}+d_{1} b_{22}\right)^{2}-4 d_{1} d_{2} \operatorname{det} \mathbf{B}, \\
\left(d_{2}\left(b_{11}+s\right)+d_{1} b_{22}\right)^{2}-4 d_{1} d_{2}\left(\operatorname{det} \mathbf{B}+s b_{22}\right)
\end{array}
$$

These terms are remarkably similar to these appearing on one of the conditions for classical Turing diffusion-driven instability evaluated on both subsystems separately. This observation supports our hypothesis at the end of Sec. I about the relation between diffusion-driven instability in the studied case and DDI conditions for patterns on $(0, \xi)$ and $(\xi, L)$.

\section{Linear stability}

With the steady state of system (6), we can analyze its stability. We focus only on the long-time behavior of the system, which can be obtained from spectral analysis, assuming that the transient behavior is not essential as shown for the classical Turing instability [35].

Let us denote the (nonconstant) steady state as $(\hat{u}, \hat{v})$ and, with a redefinition of $\tilde{u}, \tilde{v}$ to the time-dependent perturbed solution, $\tilde{u}=u-\hat{u}, \tilde{v}=v-\hat{v}$ expand the evolution equations around the steady state to find

$$
\begin{aligned}
& \partial_{t} \tilde{u}=d_{1} \partial_{x x} \tilde{u}+\left(b_{11}+h(x)\right) \tilde{u}+b_{12} \tilde{v}, \\
& \partial_{t} \tilde{v}=d_{2} \partial_{x x} \tilde{v}+b_{21} \tilde{u}+b_{22} \tilde{v} .
\end{aligned}
$$

Since we consider Neumann boundary conditions, we have a complete orthogonal basis $y_{n}(x), n \in \mathbb{N}_{0}$ of $L^{2}(0, L)$ and eigenvalues $\kappa_{n}=(n \pi / L)^{2}$ for the negative Laplacian (which satisfy $\left.-\Delta y_{n}=\kappa_{n} y_{n}\right)$. Now we rewrite functions $\tilde{u}$ and $\tilde{v}$ in terms of the series,

$$
\tilde{u}(t, x)=\sum_{n=0}^{\infty} A_{n}(t) y_{n}(x), \quad \tilde{v}(t, x)=\sum_{n=0}^{\infty} B_{n}(t) y_{n}(x) .
$$

Thus system (10) can be rewritten into the form,

$$
\sum_{n=0}^{\infty}\left(\begin{array}{l}
\partial_{t} A_{n} \\
\partial_{t} B_{n}
\end{array}\right) y_{n}(x)+\mathbf{D}\left(\begin{array}{l}
A_{n} \\
B_{n}
\end{array}\right) \kappa_{n} y_{n}(x)-\mathbf{J}(x)\left(\begin{array}{l}
A_{n} \\
B_{n}
\end{array}\right) y_{n}(x)=0,
$$

where we have introduced a standard notation,

$$
\begin{aligned}
\mathbf{D} & =\left(\begin{array}{cc}
d_{1} & 0 \\
0 & d_{2}
\end{array}\right) \\
\mathbf{J}(x) & =\left(\begin{array}{cc}
J_{11}(x) & J_{12} \\
J_{21} & J_{22}
\end{array}\right)=\left(\begin{array}{cc}
b_{11}+h(x) & b_{12} \\
b_{21} & b_{22}
\end{array}\right) .
\end{aligned}
$$

Problems of the type (12) can be solved using spectral methods, i.e., using expansions in eigenfunctions of the negative Laplacian (see Appendix A 3 for details). However, the system does not decouple into a straightforward collection of coupled ordinary differential equations for amplitudes of the eigenfunctions but rather a truncation has to be employed with detailed calculations presented in Appendix A 3, with results summarized in Appendix A 3 a. Note that extension to a general linear case with jumps in every term is straightforward (see Appendix A 4).

Note that the same qualitative behavior (with the same long-time behavior) is obtained when studying a perturbation of the piecewise constant solution (5) in a generalized function sense (see Appendix A 2), or when instead of linearizing the whole problem we linearize just the kinetics [see Appendix A 1; in particular compare Eqs. (A1) and (A14) and also compare Eqs. (A4) and (A6), noting Appendix A 4].

Although we show below that the analysis of Eq. (12) does yields correct results (via comparison to numerical solutions), this linear analysis does not allow the study of the most interesting and pertinent phenomena (being also the main motivation for this study), the influence of heterogeneity on spatial frequency of a pattern and the one-sided patterns.

\section{B. Boundary layer analysis}

To proceed with the analysis for general kinetics and to facilitate a boundary layer analysis we regularize the Heaviside replacing $h(x)$ with

$$
\begin{aligned}
h_{\delta}(x) & =\frac{s}{2}\left[1+g\left(\frac{x-\xi}{\delta}\right)\right], \quad \text { with } g \in C^{\infty}(R), \\
\lim _{x \rightarrow \pm \infty} g(x) & = \pm 1, g^{\prime} \geqslant 0,
\end{aligned}
$$

where one can think of, for example,

$$
h_{\delta}(x)=\frac{s}{2}\left[1+\tanh \left(\frac{x-\xi}{\delta}\right)\right],
$$

and consider small values of $\delta>0$. Then the steady state (assuming it exists), $\left(u_{s}, v_{s}\right)$, satisfies

$$
\begin{array}{ll}
0=d_{1} \partial_{x x} u_{s}+f\left(u_{s}, v_{s}\right)+h_{\delta}(x) u_{s}, & \\
0=d_{2} \partial_{x x} v_{s}+g\left(u_{s}, v_{s}\right) . & \text { on }(0, L) .
\end{array}
$$

Expanding about the steady state that is not designated to be a pattern, as described earlier, we have

$$
u=u_{s}+\tilde{u}, \quad v=v_{s}+\tilde{v},
$$

with

$$
\begin{aligned}
& \tilde{u}_{t}=d_{1} \partial_{x x} \tilde{u}+J_{11}\left(u_{s}, v_{s}\right) \tilde{u}+J_{12}\left(u_{s}, v_{s}\right) \tilde{v}+h_{\delta}(x) \tilde{u}, \\
& \tilde{v}_{t}=d_{2} \partial_{x x} \tilde{v}+J_{21}\left(u_{s}, v_{s}\right) \tilde{u}+J_{22}\left(u_{s}, v_{s}\right) \tilde{v}, \quad \text { on }(0, L),
\end{aligned}
$$

where $\mathbf{J}\left(u_{s}, v_{s}\right)$ is the Jacobian of the kinetics about the steady solution $\left(u_{s}, v_{s}\right)$.

We proceed with a boundary layer analysis. Based on the continuity of solution on data we argue that for small enough jump $s$ the steady-state solution that does not correspond to a pattern will be approximately $\left(\bar{u}^{L}, \bar{v}^{L}\right)$ sufficiently to the left of $x=\xi$ and approximately $\left(\bar{u}^{R}, \bar{v}^{R}\right)$ sufficiently to the right, where

$$
\begin{aligned}
f\left(\bar{u}^{L}, \bar{v}^{L}\right) & =0=g\left(\bar{u}^{L}, \bar{v}^{L}\right), \\
f\left(\bar{u}^{R}, \bar{v}^{R}\right)+s \bar{u}^{R} & =0=g\left(\bar{u}^{R}, \bar{v}^{R}\right) .
\end{aligned}
$$

Hence for $x<\xi,|x-\xi| \gg \delta$ we anticipate the approximation,

$$
\begin{aligned}
& \tilde{u}_{t}=d_{1} \partial_{x x} \tilde{u}+J_{11}\left(\bar{u}^{L}, \bar{v}^{L}\right) \tilde{u}+J_{12}\left(\bar{u}^{L}, \bar{v}^{L}\right) \tilde{v}, \\
& \tilde{v}_{t}=d_{2} \partial_{x x} \tilde{v}+J_{21}\left(\bar{u}^{L}, \bar{v}^{L}\right) \tilde{u}+J_{22}\left(\bar{u}^{L}, \bar{v}^{L}\right) \tilde{v}
\end{aligned}
$$

and, similarly,

$$
\begin{aligned}
& \tilde{u}_{t}=d_{1} \partial_{x x} \tilde{u}+J_{11}\left(\bar{u}^{R}, \bar{v}^{R}\right) \tilde{u}+J_{12}\left(\bar{u}^{R}, \bar{v}^{R}\right) \tilde{v}+s \tilde{u}, \\
& \tilde{v}_{t}=d_{2} \partial_{x x} \tilde{v}+J_{21}\left(\bar{u}^{R}, \bar{v}^{R}\right) \tilde{u}+J_{22}\left(\bar{u}^{R}, \bar{v}^{R}\right) \tilde{v},
\end{aligned}
$$


for $x>\xi,|x-\xi| \gg \delta$. These can be considered as the outer problems for a leading order boundary layer approximation.

We proceed to consider the prospects of an internal boundary layer near $x \approx \xi$. Indeed noting the form of $h_{\delta}(x)$, which drives hetergeneous behavior near $x=\xi$, one can rescale the spatial component via

$$
X=(x-\xi) / \delta
$$

This will lead to the absence of a dominant balance for an inner expansion with $\tilde{u} \sim u_{\text {in }}(X, t)+o(1)$, which instead yields $d_{1} \partial_{X X} u_{i n}=0$ at leading order, with an analogous observation for $\tilde{v} \sim v_{i n}(X, t)+o(1)$. The resulting linear solution behavior is divergent as $|X| \rightarrow \infty$ unless $u_{\text {in }}$ and $v_{\text {in }}$ are constant, indicating no boundary layer, but instead a matching of the left and right outer solutions, via a nominal but constant inner layer solution. Given the kinetics are assumed to be order unity as $\delta$ is decreased, a dominant balance at leading order, and thus more complex dynamics, is only conceivable with the concomitant temporal rescaling $T=t / \delta^{2}$. Then, at leading order,

$$
\partial_{T} u_{i n}=d_{1} \partial_{X X} u_{i n}, \quad \partial_{T} v_{i n}=d_{2} \partial_{X X} v_{i n},
$$

with the kinetics subdued by a factor of $\delta^{2}$. The resulting dynamics is very fast and, more importantly, pure diffusion. Thus it will not drive patterning within the inner region but instead instigate diffusion on a very fast time scale, acting to homogenize across the inner region, whereby for $T \gg$ 1 , i.e., $t \gg \delta^{2}$, one will expect an inner solution which is approximately constant after transients have relaxed even if the initial conditions are highly varying in the vicinity of $x \approx \xi$.

Hence, considering the impact of $\delta \ll 1$, with the limit of zero $\delta$ corresponding to the Heaviside function of interest in the kinetics, the evidence is that the inner solution of a boundary layer analysis does not induce patterning but has a rather trivial dynamics. Instead, the behavior of the outer solutions, i.e. Eqs. (16) and (17), is indicated as governing the propensity of system patterning. Proceeding, this allows one to infer that if both outer solutions are unstable, then instability on both sides of $x=\xi$ is expected. In contrast, if one outer solution is unstable and the other stable, we expect an instability on one side of $x=\xi$. Analogous reasoning suggests stability if the outer solution dynamics either side of $x=\xi$ is stable. Finally, due to this local nature of the result we expect that the spatial frequency of the emerging patterns is also related locally to the Turing conditions and hence with the prospect of a change in spatial frequency of patterning across the domain.

\section{Summary and formulation of DDI conditions}

Spectral theory as detailed in the Appendixes yields a plausible approach to stability analysis but its practical use seems to be limited as the algebraic complexity even after truncation requires a numerical approach and provides neither information about one-sided patterns nor the effect of spatial heterogeneity on the spatial frequency variation in the resulting pattern. The asymptotics, on the other hand, can be used to estimate conditions for Turing pattern emergence and its classification.

As a summary of all the above partial results suggests that the conjectured conditions are of the form of conditions for Turing's diffusively driven instability evaluated for the system with constant coefficients considered separately on intervals $(0, \xi)$ and $(\xi, L)$. Therefore let us denote the following conditions for the latter interval:

$$
\begin{aligned}
T 1^{R}:= & b_{11}+s+b_{22}<0, \\
T 2^{R}:= & \left(b_{11}+s\right) b_{22}-b_{12} b_{21}>0, \\
T 3^{R}:= & \left(b_{11}+s\right) d_{2}+b_{22} d_{1}>0, \\
T 4^{R}:= & \left(\left(b_{11}+s\right) d_{2}+b_{22} d_{1}\right)^{2}-4 d_{1} d_{2}\left(\left(b_{11}+s\right) b_{22}\right. \\
& \left.-b_{12} b_{21}\right)>0,
\end{aligned}
$$

and analogously for the former interval $T 1^{L}-T 4^{L}$ (particularly noting that $T i^{L} \equiv T i^{R}, i \in\{1, \ldots, 4\}$ with $\left.s=0\right)$. These conditions will be compared with results from a large number of numerical solutions to the full model. Further, as we discuss below, we obtain not only a very good approximation for stability conditions in practice but we also observe a strong indication that it provides a tool to distinguish among all the one-sided patterns and both-sided patterns in the case of nonlinear kinetics, which is clearly beyond the scope of the spectral analysis in the Appendixes.

In particular assuming $T 1^{L} \wedge T 2^{L} \wedge T 1^{R} \wedge T 2^{R}$ (we denote the logical and as $\wedge$ ) it could be expected that a pattern on the left will emerge only if $T 3^{L} \wedge T 4^{L}$ holds and the pattern on the right will emerge only if $T 3^{R} \wedge T 4^{R}$ holds. Therefore we propose and will subsequently numerically verify the following conditions:

$$
\begin{array}{ll}
T 1^{L} \wedge T 2^{L} \wedge T 1^{R} \wedge T 2^{R} \wedge\left(T 3^{L} \wedge T 4^{L}\right) \wedge\left(T 3^{R} \wedge T 4^{R}\right) & \text { pattern on both sides, } \\
T 1^{L} \wedge T 2^{L} \wedge T 1^{R} \wedge T 2^{R} \wedge \quad\left(T 3^{L} \wedge T 4^{L}\right) \wedge \neg\left(T 3^{R} \wedge T 4^{R}\right) & \text { pattern on the left side, } \\
T 1^{L} \wedge T 2^{L} \wedge T 1^{R} \wedge T 2^{R} \wedge \neg\left(T 3^{L} \wedge T 4^{L}\right) \wedge\left(T 3^{R} \wedge T 4^{R}\right) & \text { pattern on the right side, } \\
T 1^{L} \wedge T 2^{L} \wedge T 1^{R} \wedge T 2^{R} \wedge \neg\left(T 3^{L} \wedge T 4^{L}\right) \wedge \neg\left(T 3^{R} \wedge T 4^{R}\right) & \text { no pattern, }
\end{array}
$$

and we shall use both the spectral approach of the Appendixes and numerical solution of the full system to verify these conditions. The former can be used just for the assessment of (in)stability while the latter will be employed to check these conditions for the existence of a one-sided pattern. Note that a straightforward extension of these conditions 
TABLE I. The table summarizing the results for the regions, i.e., the sets of the parameters satisfying combinations of the conditions (18) in the explored parameter space. All parameters in each region exhibit the same behavior. We impose the following designation: $U$ and 0 denote unbounded and zero long-time solutions of the evolution problem; + and - denote signs of the largest real part of eigenvalues of the matrix (A12).

\begin{tabular}{|c|c|c|c|c|}
\hline \multicolumn{5}{|c|}{$T 1^{L} \wedge T 1^{R} \wedge T 2^{L} \wedge T 2^{R}$} \\
\hline & $T 3^{L} \wedge T 3^{R}$ & $\neg T 3^{L} \wedge T 3^{R}$ & $T 3^{L} \wedge \neg T 3^{R}$ & $\neg T 3^{L} \wedge \neg T 3^{R}$ \\
\hline $\begin{array}{l}T 4^{L} \wedge T 4^{R} \\
\neg T 4^{L} \wedge T 4^{R} \\
T 4^{L} \wedge \neg T 4^{R} \\
\neg T 4^{L} \wedge \neg T 4^{R}\end{array}$ & $\begin{array}{c}(\mathrm{U},+) \\
(\mathrm{U},+) \\
(\mathrm{U},+) \\
(0,-)\end{array}$ & $\begin{array}{c}(\mathrm{U},+) \\
(\mathrm{U},+) \\
(0,-) \\
(0,-) \\
\wedge T 1^{R} \wedge T 2^{L} \\
(\mathrm{U},+)\end{array}$ & $\begin{array}{c}(\mathrm{U},+) \\
(0,-) \\
(\mathrm{U},+) \\
(0,-)\end{array}$ & $\begin{array}{l}(0,-) \\
(0,-) \\
(0,-) \\
(0,-)\end{array}$ \\
\hline
\end{tabular}

to the general case also be implemented, as discussed in Appendix A 4.

\section{NUMERICAL APPROACH. VERIFICATION OF ESTIMATED DDI CONDITIONS}

We shall first focus on the verification of the proposed DDI conditions (18) in the linear case, where the only plausible prediction and verification is whether a small perturbation exponentially increases or decays in time. Then we proceed to test the more detailed conditions (19) in the nonlinear case where, in addition, we verify the prediction of a one-sided pattern.

\section{A. Linear case}

We have eight conditions (18) and hence 256 combinations to be analyzed. Since we are interested in a phenomenon similar to Turing's self-organization, we disregard the case when the kinetics themselves induce instability. In classical Turing patterning two of the DDI conditions are equivalent to a requirement of a stable homogeneous steady state in the absence of diffusion [36]. Therefore, in our case we assume that $T 1^{L} \wedge T 2^{L} \wedge T 1^{R} \wedge T 2^{R}$ holds [corresponding to a stable homogeneous steady state on both parts of $(0, L)]$ and we focus on the remaining 16 combinations.

The sets corresponding to each combination are denoted by distinct regions (see Table I). For all parameters in each region we want to know if a pattern can emerge or not. This crucially includes an assessment of whether one can assign this property of pattern existence to every point in each region, independent of further details. As it was noted in the previous analysis of linear system stability, we will use two tools: (i) calculating the largest real part of eigenvalues of the truncated matrix in the Appendixes, Eq. (A12), using MATLAB and (ii) solving the evolution problem (6) using MATHEMATICA (for more details on computational approaches see Appendix A 5).

In both approaches we take large sets of parameter values sampling each region. Other parameters are fixed for every numerical experiment if it is not stated otherwise. The step location is at $\xi=120$, with $L=400$, where the latter has been chosen to be sufficiently large so as not to prevent a possible pattern due to insufficient room in the domain (as checked by confirming the results are unchanged with $\xi=300, L=1000$ and $\xi=30, L=100$, for example, not shown). Further, to reduce the seven (not considering $L$ ) dimensional parameter space we fix diffusion coefficients with a sufficiently large ratio $d_{1}=1, d_{2}=100$ and also fix $s \in(-1,1)$. The remaining parametric space was discretized and the comparison between the identified conditions and computations was examined in two-dimensional slices of this parameter space and the slices with varying $b_{11}, b_{12}$ have been presented in Fig. 3 .

Before we present the relation between conditions (18) and pattern formation, we compare the numerical and spectral approaches based on computational results. First, the results from the spectral approach concur with those from solving the evolution problem. In particular, with the possible exception of the very near vicinity of the parameter space boundaries between differing stability behaviors, the largest real part of eigenvalues is negative if and only if the supremum norm of the solution to the evolution problem is smaller than the initial norm. Due to the conformity of the results from both methods while being very different conceptually the conclusions from either approximation are inferred to be generally accurate.

Second, the character of pattern emergence is indeed the same within each region from Table I. Particularly, if two regions express an opposite behavior, the change is located exactly on the border of the regions (with negligible imperfection due to numerical imprecision). Hence, these observations entail a justification of the chosen conditions (18).

The results are outlined in Table I and Fig. 3 which can be summarized as follows: A Turing pattern will emerge for large enough $\min (\xi, L-\xi)$ with unstable eigenmodes that satisfy the boundary conditions if and only if

$\left(T 1^{L} \wedge T 2^{L} \wedge T 1^{R} \wedge T 2^{R}\right) \wedge\left(\left(T 3^{L} \wedge T 4^{L}\right) \vee\left(T 3^{R} \wedge T 4^{R}\right)\right)$

holds.

In short, the hypothetical conditions (18) have been evidenced as well characterizing whether the system exhibits a pattern or not. Even though we have implemented a detailed numerical verification using two different approachescomputing a spectrum of the truncated matrix (A12) and solving the evolution problem (6) — and these conditions seem to have an intuitive explanation, we are not able to validate them nor relate them using a rigorous analytical approach, as we indicated above. However, one should bear in mind that the analysis outlined in the Appendixes via a spectral analysis is lacking rigor "only" in the limit $N \rightarrow \infty$ where 
(a)

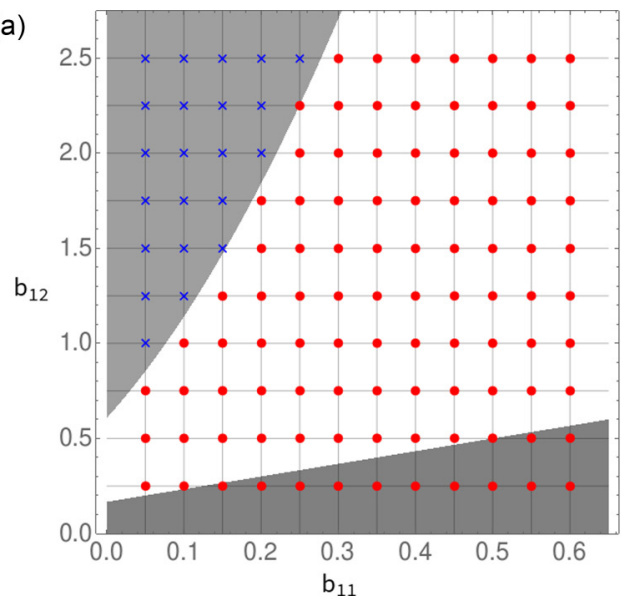

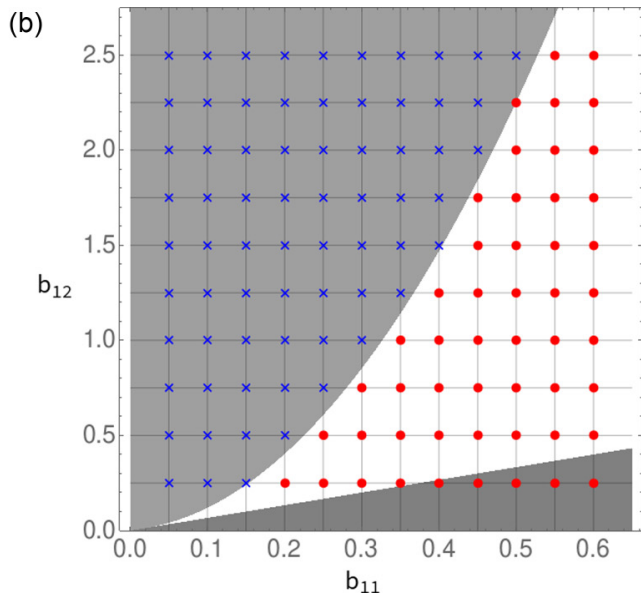

FIG. 3. An illustration of the match between the identified Turing instability conditions for affine kinetics, Eq. (20), and the results from the evolution of system (6) with $b_{10}=1, b_{20}=3, b_{21}=-3, b_{22}=-2, L=100, \xi=30, d_{1}=1, d_{2}=100$ and (a) $s=0.25$, (b) $s=-0.25$. The remaining two parameters, $b_{11}, b_{12}$, are considered as parameters for exploring stability properties and are on the $x$ and $y$ axes. In the background the conditions (20) are plotted in the grayscale, which, in increasing grayscale intensity, highlight regions with an unbounded solution indicating the existence of pattern (white), decaying solution indicating no pattern, and the region where $T 1^{L} \wedge T 2^{L} \wedge T 1^{R} \wedge T 2^{R}$ does not hold (the darkest gray). The spots denote the resulting pattern type based on numerical solution to the evolution problem: an unbounded solution indicating a pattern (a red disk) and decaying solutions corresponding to no pattern (a blue cross).

$N$ is the number of terms in the spectral expansion, while the continuum approximation motivates a finite $N$ cutoff.

\section{B. Nonlinear case}

Additionally, from (19) we have the prospect of (i) a tool to distinguish a one-sided pattern from a both-sided pattern, (ii) an indicative criterion for self-organization even for nonlinear kinetics, that is how to find conditions determining pattern emergence, and (iii) how to detect which type of the pattern it should be. We proceed to test this, considering Schnakenberg kinetics,

$$
f(u, v)=a-u+u^{2} v, \quad g(u, v)=b-u^{2} v,
$$

and Gierer-Meinhardt kinetics,

$$
f(u, v)=a-b u+\frac{u^{2}}{v}, \quad g(u, v)=u^{2}-v,
$$

with $a, b$ positive constants as two exemplars for reaction kinetics in Turing models.

Numerical experiments have been implemented using Wolfram MATHEMATICA as in the linear case (see Appendix A 5). The terminal time is $\tau=10^{3}$. This choice was sufficient to distinguish the nonexistence of pattern from its presence, where in the latter case the convergence of a norm was clearly observed suggesting a convergence of the long-time solutions to stationary patterns. The initial condition was set to be small random noise around the stationary solution $\bar{u}(x), \bar{v}(x)$. For both choices of kinetics we take $L=400, \xi=120, d_{1}=1$, $d_{2}=100$; this parameter selection follows the reasoning from the linear case. Large sets of the remaining parameters $a, b, s$ are considered to capture the rich behavior sufficiently to illustrate the legitimacy of the instability conditions (19).

In particular, the types of pattern resulting from simulations agree well with the predictions given by conditions (19) as depicted in Fig. 4. The degree of correspondence seems to be very high at least in the tested scenarios (kinetics and parameters selection) giving merit to the approach and the resulting conditions, despite the absence of rigor.

\section{CONCLUSIONS}

In this paper we considered a reaction-diffusion system with a spatial dependence via a linear kinetic term with a coefficient in the form of a spatial step function and we analyzed the resulting impact on conditions for pattern formation. First we defined a pattern as a steady solution with an inhomogeneity persevering throughout a large enough domain. Using an analytical-numerical approach we examined a case of affine kinetics and deduced conditions for pattern emergence in a very simple form, Eq. (18). For the case of nonlinear kinetics we took conditions inherited from a suitable linearization, generalizing the previous conditions to those stated in (19), with a verification for two choices of kinetics and a range of parameter values. Further, note that conditions (19) and their agreement with numerics also match the intuition of at least some experimentalists in the field, e.g., [20], and suggests further analytical progress may be feasible at least for step function behaviors in the kinetics.

If we compare our results with the previously studied system with an additive spatial step function independent of the morphogen concentrations [33], the patterns in our case have not only different amplitudes on the two sides of the step but also different frequencies. This highlights that patterning with sharp changes in spatial frequency may be a signature of kinetic heterogeneity characterized by rapid transitions in kinetics.

One interesting point is whether there is any restriction on the size of the step $s$. Although the final conditions (19) are well defined for any value of $s$, a restriction arises from our definition of a pattern in Sec. I. We need the eventual pattern to be more significant in comparison to the inhomogeneity 

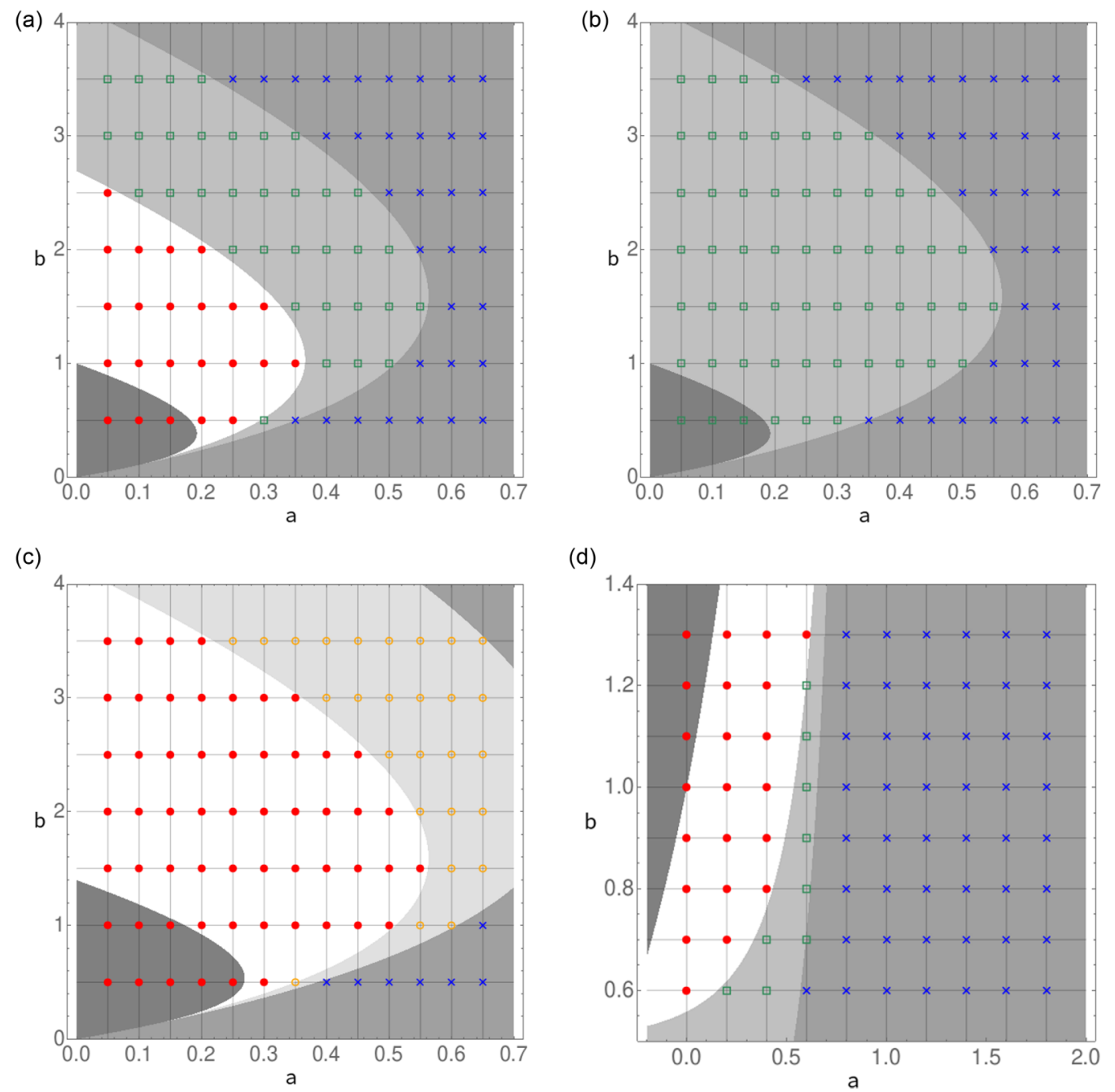

FIG. 4. An illustration of the match between the identified Turing instability conditions, Eq. (19), and the results from the evolution of system (1) with Schnakenberg kinetics (a) $s=0.25$, (b) $s=0.75$, (c) $s=-0.25$; and Gierer-Meinhardt kinetics (d) $s=0.5$. In the background the conditions (19) are plotted in the grayscale, which, in increasing grayscale intensity, highlight regions with a both-sided pattern (white), a right-sided pattern, left-sided pattern, no pattern, and the region where $T 1^{L} \wedge T 2^{L} \wedge T 1^{R} \wedge T 2^{R}$ does not hold (the darkest gray). The spots denote the resulting pattern type based on numerical solution to the evolution problem: a both-sided pattern (a red disk), a left-sided pattern (a green square), a right-sided pattern (an orange circle), and no pattern (a blue cross).

localized around $\xi$, which is expected to be true for sufficiently small $s$, by appeal to continuity with respect to parameters. For larger $s$ the localized inhomogeneity will also be larger, which can be easily seen even in the linear case due to the larger gap between $\bar{u}(x)$ [respectively, $\bar{v}(x)$ ] at the point $\xi$ and thus has every potential to invalidate our findings for sufficiently large $s$.

The conditions (19) are necessary diffusion driven instability (DDI) conditions and depend directly on the diffusion coefficients, kinetics, and the size of the step. As well as in the classical Turing system, if DDI conditions hold, a large enough domain is necessary for a pattern to emerge; thus the intervals $(0, \xi)$ and $(\xi, L)$ are also required to be sufficiently large. Moreover, sufficiently large intervals are necessary for a pattern to be correctly identifiable, as seen in the comparison of Figs. 1(b) and 2(b) where both systems are predicted to exhibit patterning on the left of the step, but only the latter does, since the subdomain $[0, \xi)$ is smaller than the emergent pattern period in Fig. 1(b). Finally, note that differences in spatial frequencies are observed to be independent of both interval lengths and hence independent of both $\xi$ and $L$. However, as the boundary layer analysis suggests, the spatial frequency can be different in the two parts of the domain as it is evaluated independently on the two subintervals.

This article is concerned with a special case of heterogeneity in the kinetics though this can be easily generalized to a certain extent. First, it is easy to see that the particular choice of a spatially dependent linear term in the kinetics is not important for the analysis even in the case of nonlinear kinetics. Further, the same approach as well as the results, will be valid for a step function $h(x)$ with finitely many steps. A limitation arises, however, due to the note from the previous paragraphs - the sizes of the steps should be small enough in comparison to the surrounding intervals so that the localized inhomogeneity due to the step does not exceed the emerging pattern in magnitude and amplitude. Further, the discreteness of eigenmodes results in a lower bound on the size of the supporting intervals of each step. 
The question of a generalization to a spatial dependency is expected for slowly varying function $h(x)$. In such a case we can take an approximation of $h(x)$ using a simple function and take the advantage of conclusions of this approximative system. In the case of a more general dependency it may be difficult to show similar conclusions. Actually, as a recent study shows [37] even shallow gradients coupled to nonlinear kinetics may lead to an unexpected and complex behavior but finding a clear-cut distinction between these cases is beyond the scope of this paper. Furthermore, how and where the present intuitive analysis fails also remains to be explored as do higher dimensional domains and curved geometries, which may allow ready generalization.

We could also consider a higher-dimensional space. The presented approach is easily extendable to domains in the form of higher-dimensional rectangles. For example, one might readily find conditions yielding the emergence of a pattern with spots on one part and stripes on the other part of a higher-dimensional domain.

Finally, let us finish with a summary of the identified hypothetical DDI conditions: (In)stability in the Turing model analyzed here appears to be a local property and can be analyzed as such, with the local assessment of whether parameters are within Turing space providing a strong indication for an unstable eigenmode excitation, at least on a sufficiently large domain with concomitant spatial frequency heterogeneity.

\section{ACKNOWLEDGMENTS}

We are grateful to our colleague Andrew Krause for providing valuable comments on our manuscript. V.K. is grateful for support from the International Mobility of Researchers-MSCA-IF in Czech Technical University, Grant No. CZ.02.2.69/0.0/0.0/17_050/0008025, funded by The Ministry of Education, Youth and Sports (MEYS) of the Czech Republic, European Regional Development Fund Project "Center for Advanced Applied Science" (Project No. CZ.02.1.01/0.0/0.0/16 019/0000778), and the Mathematical Institute at the University of Oxford.

\section{APPENDIX: FURTHER DETAILS OF STABILITY ANALYSIS}

In the main text, Sec. II A 2, we follow a rather standard approach to linear stability analysis and simply adopt it for a system with a jump in reaction kinetics. Here, for the sake of completeness, we pursue other possible approaches and, as we shall show, all entail the same qualitative behavior. Namely, we expand the jump in a generalized function sense (see Appendix A 2), and linearize just the kinetics instead of linearization of the whole problem (see Appendix A 1).

These approaches lead to the problem of the type in Eq. (12) which can be further analyzed using spectral methods, i.e., using expansions in eigenfunctions of negative Laplacian (see Appendix A 3 for details). However, the system does not decouple into a straightforward collection of coupled ordinary differential equations for amplitudes of the eigenfunctions but rather a truncation has to be employed. Finally, an extension to a general linear case with jumps in every term is straightforward as noted in Appendix A 4. Note that all computational results shown in this work were obtained via two approaches which are described in Appendix A 5.

\section{Alternative I. Linearization of kinetics only}

An alternative approach is to linearize only the kinetics around the piecewise constant naive steady state.

Assume the existence of a solution $\left(\bar{u}^{L}, \bar{v}^{L}\right)$ and $\left(\bar{u}^{R}, \bar{v}^{R}\right)$ satisfying

$$
\begin{aligned}
f\left(\bar{u}^{L}, \bar{v}^{L}\right) & =0=g\left(\bar{u}^{L}, \bar{v}^{L}\right), \\
f\left(\bar{u}^{R}, \bar{v}^{R}\right)+s \bar{u}^{R} & =0=g\left(\bar{u}^{R}, \bar{v}^{R}\right),
\end{aligned}
$$

and take approximations of reaction kinetics as Taylor expansions evaluated separately on both intervals $(\tilde{u}=u-\bar{u})$ :

$$
\begin{aligned}
L: f(u, v) & =f\left(\bar{u}^{L}, \bar{v}^{L}\right)+J_{11}^{L} \tilde{u}+J_{12}^{L} \tilde{v}+\ldots \\
g(u, v) & =g\left(\bar{u}^{L}, \bar{v}^{L}\right)+J_{21}^{L} \tilde{u}+J_{22}^{L} \tilde{v}+\ldots, \\
R: f(u, v)+s u & =f\left(\bar{u}^{R}, \bar{v}^{R}\right)+s \bar{u}^{R}+J_{11}^{R} \tilde{u}+J_{12}^{R} \tilde{v}+\ldots \\
g(u, v) & =g\left(\bar{u}^{R}, \bar{v}^{R}\right)+J_{21}^{R} \tilde{u}+J_{22}^{R} \tilde{v}+\ldots,
\end{aligned}
$$

where $J_{i j}^{L}$ (respectively, $J_{i j}^{R}$ ) denote the elements of the Jacobian matrix of the map $(f, g)$ at $\left(\bar{u}^{L}, \bar{v}^{L}\right)$ [respectively, $(f+s u, g)$ at $\left.\left(\bar{u}^{R}, \bar{v}^{R}\right)\right]$. Using the following notation,

$$
\begin{aligned}
J_{i j}(x) & =\left\{\begin{array}{ll}
J_{i j}^{L} & x \in(0, \xi) \\
J_{i j}^{R} & x \in(\xi, L)
\end{array}, \quad \bar{u}(x)=\left\{\begin{array}{ll}
\bar{u}^{L} & x \in(0, \xi) \\
\bar{u}^{R} & x \in(\xi, L)
\end{array},\right.\right. \\
\bar{v}(x) & =\left\{\begin{array}{ll}
\bar{v}^{L} & x \in(0, \xi) \\
\bar{v}^{R} & x \in(\xi, L)
\end{array},\right.
\end{aligned}
$$

we can write down an affine system describing evolution around $\bar{u}(x), \bar{v}(x)$ while approximating the original system with nonlinear kinetics as

$$
\begin{aligned}
& \partial_{t} u=d_{1} \partial_{x x} u+J_{11}(x) u+J_{12}(x) v+c_{1}(x), \\
& \partial_{t} v=d_{2} \partial_{x x} v+J_{21}(x) u+J_{22}(x) v+c_{2}(x),
\end{aligned}
$$

with the step functions,

$$
\begin{aligned}
& c_{1}(x)=-J_{11}(x) \bar{u}(x)-J_{12}(x) \bar{v}(x), \\
& c_{2}(x)=-J_{21}(x) \bar{u}(x)-J_{22}(x) \bar{v}(x) .
\end{aligned}
$$

The analysis of such a system in the current framework is discussed in Appendix A 4.

\section{Alternative II. Linearization about a piecewise constant steady state}

The second option could be to linearize around step functions $(\bar{u}, \bar{v})$. If we try to proceed, we will obtain a linear system, but with terms containing a derivative of the Dirac delta function, which results from the nontrivial step in $(\bar{u}, \bar{v})$. Therefore, we are not able to obtain the linearized system following the standard approach above.

However, it is instructive to proceed further as an expansion (in generalized functions) of the delta function in the eigenfunctions $\left\{y_{k}\right\}$ is available and hence we can rewrite the 
linearized system yet again in terms of a system of equations for particular modes.

Linearization of the system (1) around step functions $(\bar{u}(x), \bar{v}(x))$ [defined in (5)] is well defined in the distributional sense and is of the form,

$$
\begin{aligned}
& \partial_{t} \tilde{u}=d_{u} \partial_{x x} \tilde{u}+b_{11} \tilde{u}+b_{12} \tilde{v}+s_{u} d_{u} \delta^{\prime}(x-\xi) \\
& \partial_{t} \tilde{v}=d_{v} \partial_{x x} \tilde{v}+b_{21} \tilde{u}+b_{22} \tilde{v}+s_{v} d_{v} \delta^{\prime}(x-\xi),
\end{aligned} \quad \text { in }(0, L),
$$

where $\left(s_{u}, s_{v}\right)$ denotes the sizes of the step of $(\bar{u}(x), \bar{v}(x))$ at $\xi, \delta(x)$ denotes Dirac delta function and $u(x)=\tilde{u}(x)-\bar{u}(x)$, $v(x)=\tilde{v}(x)-\bar{v}(x)$.

Since Neumann boundary conditions are considered, we expand $(\tilde{u}, \tilde{v})$ using orthonormal basis $\left\{y_{n}\right\}_{n \in\{0,1, . .\}}=$
$\left\{\frac{1}{L}, \frac{2}{L} \cos \left(\frac{n \pi}{L} x\right)\right\}_{n=1}^{\infty}$ as the series,

$$
\tilde{u}=\sum_{n=0}^{\infty} A_{n} y_{n}, \quad \tilde{v}=\sum_{n=0}^{\infty} B_{n} y_{n}
$$

and rewrite system (A2) in the form of a system of equations for each eigenmode. The Dirac delta function can be expanded in terms of any eigenfunctions of the Laplacian on any interval. Hence we use the following expansion of Dirac delta function on $(0, L)$ :

$$
\delta(x-\xi)=\frac{2}{L} \sum_{n=1}^{\infty} \sin \left(\frac{n \pi \xi}{L}\right) \sin \left(\frac{n \pi x}{L}\right) .
$$

Therefore the linearized problem has the following eigenmode expansion:

$$
\sum_{n=1}^{\infty} y_{n}\{\left(\begin{array}{c}
\dot{A}_{n} \\
\dot{B}_{n}
\end{array}\right)+\left[\left(\begin{array}{cc}
d_{u} & 0 \\
0 & d_{v}
\end{array}\right) \kappa_{n}-\mathbf{J}(x)\right]\left(\begin{array}{l}
A_{n} \\
B_{n}
\end{array}\right)-\underbrace{\frac{n \pi}{L} \sin \left(\frac{n \pi \xi}{L}\right)\left(\begin{array}{cc}
d_{u} s_{u} & 0 \\
0 & d_{v} s_{v}
\end{array}\right)\left(\begin{array}{l}
A_{n} \\
B_{n}
\end{array}\right)}_{\text {forcing }}\}=0
$$

where $\kappa_{n}=\left(\frac{n \pi}{L}\right)^{2}$ and the matrix of linearized kinetics $\mathbf{J}(x)$ is evaluated at the piecewise constant function $(\bar{u}(x), \bar{v}(x))$. As the $\delta^{\prime}(x-\xi)$ contribution translates only into a (constant) forcing, it does not affect the (in)stability result. Then the generalized function approach yields exactly the same problem as previously derived in Eq. (12).

\section{Detailed analysis of the dispersion relation}

The difference from the standard Turing system analysis for a homogeneous system emerges from the spatial dependence of $\mathbf{J}(x)$ preventing the decoupling of individual eigenmodes and hence preventing a straightforward solution. However, we can take the advantage of the fact that $\mathbf{J}(x)$ contains only constants and a step function, all satisfying Neumann boundary conditions and hence within the span of the eigenfunctions $\left\{y_{k}\right\}$,

$$
\mathbf{J}(x)=\sum_{k=0}^{\infty} \underbrace{\left(\begin{array}{cc}
J_{11}^{(k)} & J_{12}^{(k)} \\
J_{21}^{(k)} & J_{22}^{(k)}
\end{array}\right)}_{\mathbf{J}^{(k)}} y_{k}(x) .
$$

The system can be rewritten as

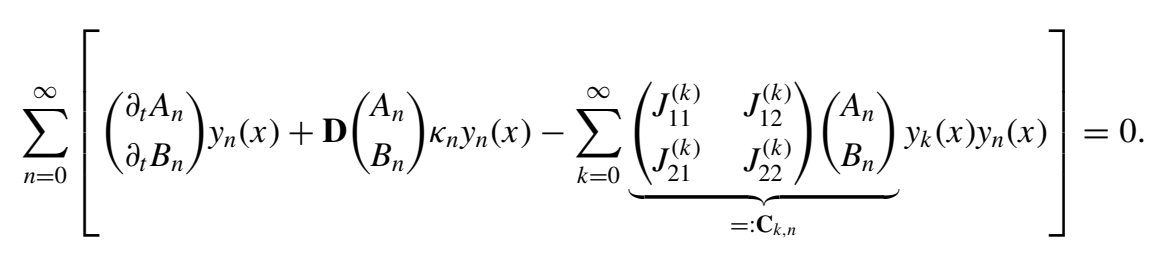

The eigenfunctions of the negative Laplacian on a one-dimensional interval are of the well-known form $y_{n}(x)=\cos (n \pi x / L)$ and hence we have

$$
y_{k}(x) y_{n}(x)=\frac{1}{2}\left(\cos \frac{(n+k) \pi x}{L}+\cos \frac{(n-k) \pi x}{L}\right)=\frac{1}{2}\left(\cos \frac{(n+k) \pi x}{L}+\cos \frac{|n-k| \pi x}{L}\right)=\frac{y_{n+k}(x)+y_{|n-k|}(x)}{2},
$$

which are again functions from the orthogonal basis. To obtain the dispersion relation we need to reorder the second sum to be able to factor out the function $y_{n}(x)$ and then invoke orthogonality of the orthogonal basis to transform the problem into an infinite system of ordinary differential equations. Denoting the coefficients in the internal sum by $\mathbf{C}_{k, n} \in \mathbb{R}^{2}$ we obtain the following form of the system:

$$
\sum_{m=0}^{\infty}\left(\begin{array}{l}
\partial_{t} A_{m} \\
\partial_{t} B_{m}
\end{array}\right) y_{m}(x)+\mathbf{D}\left(\begin{array}{l}
A_{m} \\
B_{m}
\end{array}\right) \kappa_{m} y_{m}(x)=\frac{1}{2} \sum_{m=0}^{\infty}\left(\sum_{n=0}^{m} \mathbf{C}_{m-n, n}+\sum_{n=m}^{\infty} \mathbf{C}_{n-m, n}\right) y_{m}(x)+\frac{1}{2} \sum_{m=1}^{\infty} \sum_{n=0}^{\infty} \mathbf{C}_{n+m, n} y_{m}(x) .
$$


The coupled evolution equations for the eigenmodes are then of the form,

$$
\begin{aligned}
0 & =\left(\begin{array}{l}
\partial_{t} A_{m} \\
\partial_{t} B_{m}
\end{array}\right)+\mathbf{D}\left(\begin{array}{l}
A_{m} \\
B_{m}
\end{array}\right) \kappa_{m}-\frac{1}{2} \sum_{n=0}^{\infty} \mathbf{C}_{|m-n|, n}-\frac{1}{2} \sum_{n=0}^{\infty} \mathbf{C}_{m+n, n}-\frac{1}{2} \mathbf{C}_{0, m}, \quad \text { for } m \geqslant 1, \\
0 & =\left(\begin{array}{l}
\partial_{t} A_{0} \\
\partial_{t} B_{0}
\end{array}\right)+\mathbf{D}\left(\begin{array}{l}
A_{0} \\
B_{0}
\end{array}\right) \kappa_{0}-\frac{1}{2} \sum_{n=0}^{\infty} \mathbf{C}_{n, n}-\frac{1}{2} \mathbf{C}_{0,0} .
\end{aligned}
$$

In our case, the elements of matrices $\mathbf{J}^{(k)}$ can be computed as

$$
\mathbf{J}^{(k)}=\left(\begin{array}{ll}
J_{11}^{(k)} & J_{12}^{(k)} \\
J_{21}^{(k)} & J_{22}^{(k)}
\end{array}\right)= \begin{cases}\left(\begin{array}{cc}
Z_{k} & 0 \\
0 & 0
\end{array}\right) & k \geqslant 1 \\
\left(\begin{array}{cc}
Z_{0} & b_{21} \\
b_{21} & b_{22}
\end{array}\right) & k=0,\end{cases}
$$

where the spatial heterogeneity is represented by a step function and where

$$
\begin{aligned}
& Z_{k}=\frac{\left\langle h(x), y_{k}(x)\right\rangle}{\left\|y_{k}(x)\right\|^{2}}=\frac{2}{L} \int_{\xi}^{L} s \cos \frac{k \pi x}{L} d x=-\frac{2 s}{k \pi} \sin \frac{k \pi \xi}{L}, \\
& Z_{0}=\frac{\left\langle h(x)+b_{11}, y_{0}(x)\right\rangle}{\left\|y_{0}(x)\right\|^{2}}=b_{11}+\frac{1}{L}\langle h(x), 1\rangle=b_{11}+\frac{s(L-\xi)}{L} .
\end{aligned}
$$

While in the case of spatial homogeneity, the spectrum and dispersion relation for the system rate of growth in terms of the wave number is given by the solvability condition for the eigenmodes, the analogous information is not analytically accessible in this framework for spatially heterogeneous functions.

Nevertheless, the system (A9) is linear and hence the solution can be written in terms of an exponential of a linear operator. Since we are not able to calculate the spectrum of the infinite matrix,

$$
\left(\begin{array}{ccccccc}
Z_{0}-d_{1} \kappa_{0} & b_{12} & \frac{Z_{1}}{2} & 0 & \frac{Z_{2}}{2} & 0 & \ldots \\
b_{21} & b_{22}-d_{2} \kappa_{0} & 0 & 0 & 0 & 0 & \ldots \\
Z_{1} & 0 & Z_{0}+\frac{Z_{2}}{2}-d_{1} \kappa_{1} & b_{12} & \frac{Z_{1}+Z_{3}}{2} & 0 & \ldots \\
0 & 0 & b_{21} & b_{22}-d_{2} \kappa_{1} & 0 & 0 & \ldots \\
Z_{2} & 0 & \frac{Z_{1}+Z_{3}}{2} & 0 & Z_{0}+\frac{Z_{4}}{2}-d_{1} \kappa_{2} & b_{12} & \ldots \\
0 & 0 & 0 & 0 & b_{21} & b_{22}-d_{2} \kappa_{2} & \ldots \\
Z_{3} & 0 & \frac{Z_{2}+Z_{4}}{2} & 0 & \frac{Z_{1}+Z_{5}}{2} & 0 & \ldots \\
0 & 0 & 0 & 0 & 0 & 0 & \ldots \\
\vdots & \vdots & \vdots & \vdots & \vdots & \vdots & \ddots
\end{array}\right),
$$

we will use MATLAB to estimate it by calculating spectrum of its truncated principal submatrix $\mathbf{M}_{n} \in \mathbb{C}^{2 n, 2 n}$. First let us explore some properties of the infinite matrix. It can be rewritten via the following synoptic sum of two matrices,

$$
\left(\begin{array}{cccccc}
0 & \frac{Z_{1}}{2} & \frac{Z_{2}}{2} & \frac{Z_{3}}{2} & \frac{Z_{4}}{2} & \ldots \\
Z_{1} & \frac{Z_{2}}{2} & \frac{Z_{1}+Z_{3}}{2} & \frac{Z_{2}+Z_{4}}{2} & \frac{Z_{3}+Z_{5}}{2} & \ldots \\
Z_{2} & \frac{Z_{1}+Z_{3}}{2} & \frac{Z_{4}}{2} & \frac{Z_{1}+Z_{5}}{2} & \frac{Z_{2}+Z_{6}}{2} & \ldots \\
Z_{3} & \frac{Z_{2}+Z_{4}}{2} & \frac{Z_{1}+Z_{5}}{2} & \frac{Z_{6}}{2} & \frac{Z_{1}+Z_{7}}{2} & \ldots \\
Z_{4} & \frac{Z_{3}+Z_{5}}{2} & \frac{Z_{2}+Z_{6}}{2} & \frac{Z_{1}+Z_{7}}{2} & \frac{Z_{8}}{2} & \ldots \\
\vdots & \vdots & \vdots & \vdots & \vdots & \ddots
\end{array}\right) \otimes\left(\begin{array}{cc}
1 & 0 \\
0 & 0
\end{array}\right)+\bigoplus_{i=0}^{\infty}\left(\begin{array}{cc}
Z_{0}-d_{1} \kappa_{i} & b_{12} \\
b_{21} & b_{22}-d_{2} \kappa_{i}
\end{array}\right),
$$

where $\otimes$ stands for the Kronecker product and $\oplus$ denotes a direct sum.

Since $Z_{i}$ denotes a Fourier coefficient and its norm vanishes for $i \rightarrow \infty$, the first matrix is bounded, compact, and has a high degree of symmetry and therefore can be intuitively understood as a small perturbation of the second matrix which is unbounded since $\kappa_{i}$ grows to infinity as $i \rightarrow \infty$. We will not be able to show that the spectrum of the infinite matrix is a limit of the spectrum of the truncated matrices but we shall show that the stability of the truncated linear system is determined by spectrum of a matrix of a relatively small dimension. Further we may justify truncating the matrix due to the continuum approximation, which is behind the formulation of the model itself. 


\section{a. Spectra of truncated matrices}

We shall show that with $\sigma\left(\mathbf{M}_{N+1}\right)$ denoting the spectrum of the truncated matrix $\mathbf{M}_{N+1}$,

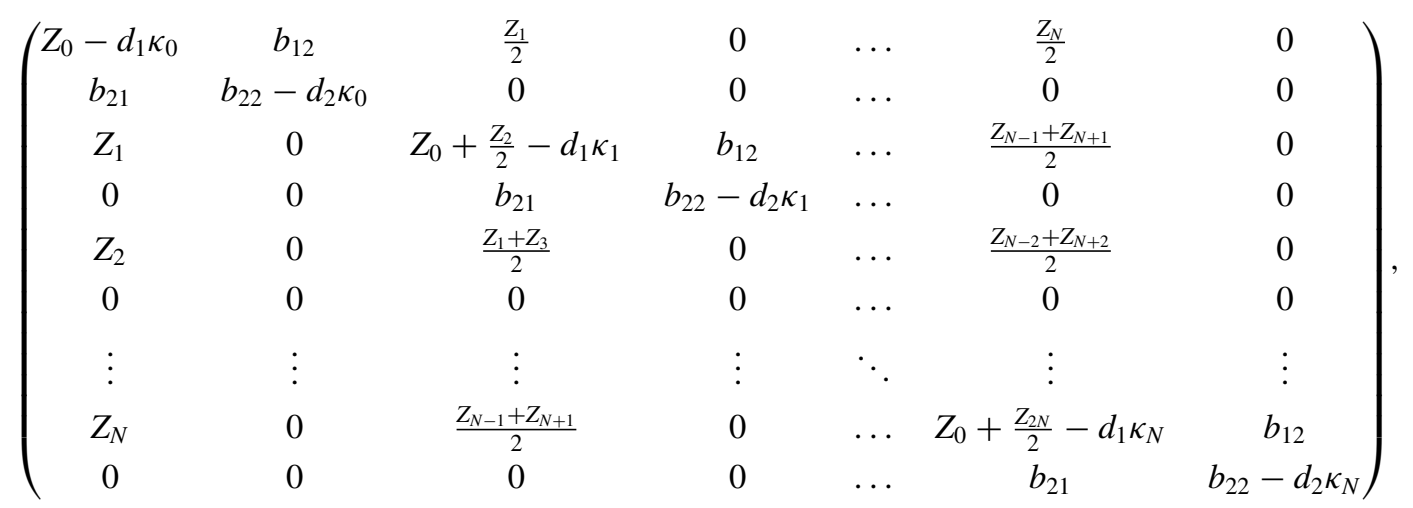

it holds that $\sigma\left(\mathbf{M}_{N+1}\right) \approx \sigma\left(\mathbf{M}_{N}\right) \cup\left\{-d_{1} \kappa_{N}+O(1),-d_{2} \kappa_{N}+O(1)\right\}$ as $N \rightarrow \infty$.

In particular, we shall show that with $N$ large enough, two eigenvalues are of the order $\kappa_{N}$ (which grows to infinity as $N \rightarrow \infty$ ). With $\lambda=\mu \kappa_{N}, \mu=O(1)$ as $N \rightarrow \infty$ we have that

$$
\begin{aligned}
\operatorname{det}\left(\mathbf{M}_{N+1}-\lambda \mathbf{I}\right) & =\kappa_{N}^{2}\left\{\left[\operatorname{det}\left(\mathbf{M}_{N}-\lambda \mathbf{I}\right)\left(\frac{1}{\kappa_{N}}\left(Z_{0}+Z_{2 N} / 2\right)-d_{1}-\mu\right)+O\left(\kappa_{N}\right)^{2 N-1}\right]\left(\frac{b_{22}}{\kappa_{N}}-d_{2}-\mu\right)+\frac{b_{12}}{\kappa_{N}} O\left(\kappa_{N}\right)^{2 N}\right\} \\
& =\operatorname{det}\left(\mathbf{M}_{N}-\lambda \mathbf{I}\right)\left(b_{22}-\kappa_{N}\left(d_{2}+\mu\right)\right)\left(Z_{0}+\frac{Z_{2 N}}{2}-\kappa_{N}\left(d_{1}+\mu\right)\right)+O\left(\kappa_{N}\right)^{2 N+1}
\end{aligned}
$$

where we note that the $\operatorname{det}\left(\mathbf{M}_{N}\right)$ is a polynomial of $2 N$ th order. Therefore two eigenvalues are indeed of the order $\kappa_{N}$, in particular $\lambda_{1}=-d_{1} \kappa_{N}+O(1)$ and $\lambda=-d_{2} \kappa_{N}+O(1)$, while the remaining $2 N$ eigenvalues are (in the leading order) the eigenvalues of $\mathbf{M}_{N}$. Hence the information about the stability associated with an arbitrarily large truncated matrix can be deduced from a smaller matrix $\mathbf{M}_{N}$ (in practice the choice of $N=50$ seems to be good enough). In addition, we anticipate that this characteristic of the spectrum will translate even into the arbitrarily large $N$ case and which seems to be confirmed by the numerical calculations in the main text.

\section{Spatial dependence in other kinetics coefficients}

We now consider the linear system (6) but with spatial dependence in every kinetic coefficient. This dependence is in the form of a step function with various step sizes but located at the same point $\xi$. Thus consider

$$
\begin{array}{ll}
\partial_{t} u=d_{1} \partial_{x x} u+b_{10}(x)+b_{11}(x) u+b_{12}(x) v, & \text { in }(0, L), \\
\partial_{t} v=d_{2} \partial_{x x} v+b_{20}(x)+b_{21}(x) u+b_{22}(x) v, & \text { in }(0, L),
\end{array}
$$

with

$$
b_{i j}(x)=\left\{\begin{array}{ll}
b_{i j} & x \in[0, \xi) \\
b_{i j}+s_{i j} & x \in[\xi, L]
\end{array} \quad i \in\{1,2\}, j \in\{0,1,2\},\right.
$$

and we assert that the procedure and conclusion are the same as in the above. Indeed, let us briefly repeat the procedure. The system (A14) has constant coefficients if considered separately on intervals $(0, \xi)$ and $(\xi, L)$, therefore the steady state can be expressed at least in principle. The stability analysis proceeds in the same way, only the Fourier coefficients $\mathbf{J}^{(k)}$,
Eq. (A10), are of the form,

$$
\begin{aligned}
& J_{i j}^{(k)}=\frac{\left\langle h(x), y_{k}(x)\right\rangle}{\left\|y_{k}(x)\right\|^{2}}=\frac{2}{L} \int_{\xi}^{L} s_{i j} \cos \frac{k \pi x}{L} d x=-\frac{2 s_{i j}}{k \pi} \sin \frac{k \pi \xi}{L}, \\
& J_{i j}^{(0)}=\frac{\left\langle b_{i j}(x), y_{0}(x)\right\rangle}{\left\|y_{0}(x)\right\|^{2}}=b_{i j}+\frac{s_{i j}(L-\xi)}{L},
\end{aligned}
$$

and the stability matrix,

$$
\left.\begin{array}{cccccc}
0 & \frac{\mathbf{J}^{(1)}}{2} & \frac{\mathbf{J}^{(2)}}{2} & \frac{\mathbf{J}^{(3)}}{2} & \frac{\mathbf{J}^{(4)}}{2} & \ldots \\
\mathbf{J}^{(1)} & \frac{\mathbf{J}^{(2)}}{2} & \frac{\mathbf{J}^{(1)}+\mathbf{J}^{(3)}}{2} & \frac{\mathbf{J}^{(2)}+\mathbf{J}^{(4)}}{2} & \frac{\mathbf{J}^{(3)}+\mathbf{J}^{(5)}}{2} & \ldots \\
\mathbf{J}^{(2)} & \frac{\mathbf{J}^{(1)}+\mathbf{J}^{(3)}}{2} & \frac{\mathbf{J}^{(4)}}{2} & \frac{\mathbf{J}^{(1)}+\mathbf{J}^{(5)}}{2} & \frac{\mathbf{J}^{(2)}+\mathbf{J}^{(6)}}{2} & \ldots \\
\mathbf{J}^{(3)} & \frac{\mathbf{J}^{(2)}+\mathbf{J}^{(4)}}{2} & \frac{\mathbf{J}^{(1)}+\mathbf{J}^{(5)}}{2} & \frac{\mathbf{J}^{(6)}}{2} & \frac{\mathbf{J}^{(1)}+\mathbf{J}^{(7)}}{2} & \ldots \\
\mathbf{J}^{(4)} & \frac{\mathbf{J}^{(3)}+\mathbf{J}^{(5)}}{2} & \frac{\mathbf{J}^{(2)}+\mathbf{J}^{(6)}}{2} & \frac{\mathbf{J}^{(1)}+\mathbf{J}^{(7)}}{2} & \frac{\mathbf{J}^{(8)}}{2} & \ldots \\
\vdots & \vdots & \vdots & \vdots & \vdots & \ddots
\end{array}\right)
$$

does not have zero elements in general $\left(\mathbf{J}^{(k)}\right.$ denotes $2 \times 2$ matrices), although analogous qualitative properties still hold. The appropriate conditions are constructed following the same idea:

$$
\begin{aligned}
T 1^{R}:= & b_{11}+s_{11}+b_{22}+s_{22}<0, \\
T 2^{R}:= & \left(b_{11}+s_{11}\right)\left(b_{22}+s_{22}\right)-\left(b_{12}+s_{12}\right)\left(b_{21}+s_{21}\right)>0, \\
T 3^{R}:= & \left(b_{11}+s_{11}\right) d_{2}+\left(b_{22}+s_{22}\right) d_{1}>0, \\
T 4^{R}:= & \left(\left(b_{11}+s_{11}\right) d_{2}+\left(b_{22}+s_{22}\right) d_{1}\right)^{2} \\
& -4 d_{1} d_{2}\left(\left(b_{11}+s_{11}\right)\left(b_{22}+s_{22}\right)\right. \\
& \left.-\left(b_{12}+s_{12}\right)\left(b_{21}+s_{21}\right)\right)>0,
\end{aligned}
$$


with $T 1^{L}-T 4^{L}$ of exactly the same form (i.e., $T 1^{R}-T 4^{R}$ with $\left.s_{i j} \equiv 0\right)$. The hypothesized conditions for pattern formation are of the same form as in Eq. (20) and, as in the former case, were verified using both numerical studies of the model equations and by analyzing the eigenvalues of truncated matrices from the spectral theory (results not shown).

\section{Computational approaches}

The spectrum of the truncated matrix (A12) is computed using the MATLAB inbuilt function eig. When denoting $N$ as a constant representing the size of matrix $[(2 N+2) \times$ $(2 N+2)]$ our numerical results show that for $N>50$ the value of the largest real part of the eigenvalues does not significantly change; larger matrices contribute to the spectrum by eigenvalues with larger negative part as we discussed above. We choose $N=1000$. With constant $M$ representing the truncation in eigenmode expansion of $h(x)$, i.e., we approximate

$$
h(x) \approx \sum_{k=0}^{M} Z^{k} y_{k}(x), \quad \text { where }\left|Z^{k}\right| \lesssim \frac{s}{k} .
$$

However, the length of numerical calculation does not significantly increase with larger $M$, so we set $M=N$.

The solutions of the evolution system (6) are computed by Wolfram MATHEMATICA 10 using NDSolve (via the method of lines for the temporal discretization and finite differences for space) up to time $\tau=10^{3}$ or until the supremum norm of the solution exceeds $10^{7}$. The initial condition is random noise, uniformly distributed between $\left(10^{-2}, 10^{2}\right)$.

Both approaches to assess stability should yield the same result as they describe the same process. However, both methods are approximate and hence small differences might occur especially close to the border of the parameter regions due to different accuracy of the approximation (truncation of the matrix versus numerical discretization when computing the evolution problem).
[1] A. M. Turing, The chemical basis of morphogenesis, Philos. Trans. R. Soc. London B 237, 37 (1952).

[2] P. K. Maini, R. E. Baker, and C.-M. Chuong, The Turing model comes of molecular age, Science 314, 1397 (2006).

[3] M. A. J. Chaplain, M. Ganesh, and I. G. Graham, Spatiotemporal pattern formation on spherical surfaces: numerical simulation and application to solid tumour growth, J. Math. Biol. 42, 387 (2001).

[4] V. Klika, R. E. Baker, D. Headon, and E. A. Gaffney, The influence of receptor-mediated interactions on reaction-diffusion mechanisms of cellular self-organisation, Bull. Math. Biol. 74, 935 (2012).

[5] J. D. Glover, K. L. Wells, F. Matthäus, K. J. Painter, W. Ho, J. Riddell, J. A. Johansson, M. J. Ford, C. A. B. Jahoda, V. Klika, R. L. Mort, and D. J. Headon, Hierarchical patterning modes orchestrate hair follicle morphogenesis, PloS Biol. 15, e2002117 (2017).

[6] A. Nakamasu, G. O. Takahashi, A. Kanbe, and S. Kondo, Interactions between zebrafish pigment cells responsible for the generation of Turing patterns, PNAS 106, 8429 (2009).

[7] S. Pantalacci, J. Prochazka, A. Martin, M. Rothova, A. Lambert, L. Bernard, C. Charles, L. Viriot, R. Peterkova, and V. Laudet, Patterning of palatal rugae through sequential addition reveals an anterior/posterior boundary in palatal development, BMC Dev. Biol. 8, 1 (2008).

[8] R. Sheth, L. Marcon, M. F. Bastida, M. Junco, L. Quintana, R. Dahn, M. Kmita, J. Sharpe, and M. A. Ros, Hox genes regulate digit patterning by controlling the wavelength of a Turing-type mechanism, Science 338, 1476 (2012).

[9] S. Kondo and T. Miura, Reaction-diffusion model as a framework for understanding biological pattern formation, Science 329, 1616 (2010).

[10] A. D. Economou, A. Ohazama, T. Porntaveetus, P. T. Sharpe, S. Kondo, A. M. Basson, A. Gritli-Linde, M. T. Cobourne, and J. B. A. Green, Periodic stripe formation by a Turing mechanism operating at growth zones in the mammalian palate, Nat. Genet. 44, 348 (2012).
[11] J. Raspopovic, L. Marcon, L. Russo, and J. Sharpe, Digit patterning is controlled by a Bmp-Sox9-Wnt Turing network modulated by morphogen gradients, Science 345, 266 (2014).

[12] L. Wolpert, Positional information and the spatial pattern of cellular differentiation, J. Theor. Biol. 25, 1 (1969).

[13] J. B. A. Green and J. Sharpe, Positional information and reaction-diffusion: Two big ideas in developmental biology combine, Development 142, 1203 (2015).

[14] T. Miura, Turing and Wopert work together during limb development, Science Signaling 6, 1 (2013).

[15] A. D. Economou and J. B. A. Green, Thick and thin fingers point out Turing waves, Genome Biology 14, 101 (2013).

[16] A. Gierer and H. Meinhardt, A theory of biological pattern formation, Kybernetik 12, 30 (1972).

[17] J. Schnakenberg, Simple chemical reaction systems with limit cycle behavior, J. Theor. Biol. 81, 389 (1979).

[18] D. Thomas, Artificial enzyme membranes, transport, memory and oscillatory phenomena, in Analysis and Control of Immobilized Enzyme Systems, edited by D. Thomas and J. P. Kernevez (North-Holland/Elsevier, Amsterdam/New York, 1976), pp. 115-150.

[19] J. D. Murray, Mathematical Biology. II Spatial Models and Biomedical Applications (Springer-Verlag, New York, 2001).

[20] D. G. Míguez, M. Dolnik, A. P. Munuzuri, and L. Kramer, Effect of Axial Growth on Turing Pattern Formation, Phys. Rev. Lett. 96, 048304 (2006).

[21] K. J. Painter, G. S. Hunt, K. L. Wells, J. A. Johansson, and D. J. Headon, Towards an integrated experimental-theoretical approach for assessing the mechanistic basis of hair and feather morphogenesis, Interface Focus 2, 433 (2012).

[22] K. M. Page, P. K. Maini, and N. A. M. Monk, Complex pattern formation in reaction-diffusion systems with spatially varying parameters, Physica D: Nonlinear Phenomena 202, 95 (2005).

[23] S. T. A. Pickett and M. L. Cadenasso, Landscape ecology: Spatial heterogeneity in ecological systems, Science 269, 331 (1995). 
[24] A. Warmflash, B. Sorre, F. Etoc, E. D. Siggia, and A. H. Brivanlou, A method to recapitulate early embryonic spatial patterning in human stem cells, Nat. Methods 11, 847 (2014).

[25] D. Iron and M. J. Ward, Spike pinning for the Gierer-Meinhardt model, Math. Comp. Simul. 55, 419 (2001).

[26] J. Wei and M. Winter, Spikes for the Gierer-Meinhardt system with discontinuous diffusion coefficients, J. Nonlinear Sci. 19, 301 (2009).

[27] M. J. Ward, D. McInerney, P. Houston, D. Gavaghan, and P. Maini, The dynamics and pinning of a spike for a reactiondiffusion system, SIAM J. Appl. Math. 62, 1297 (2002).

[28] D. L. Benson, J. A. Sherratt, and P. K. Maini, Diffusion driven instability in an inhomogeneous domain, Bull. Math. Biol. 55, 365 (1993).

[29] T. Glimm, J. Zhang, and Y.-Q. Shen, Interaction of Turing patterns with an external linear morphogen gradient, Nonlinearity 22, 2541 (2009).

[30] T. Glimm, J. Zhang, Y.-Q. Shen, and S. A. Newman, Reactiondiffusion systems and external morphogen gradients: The twodimensional case, with an application to skeletal pattern formation, Bull. Math. Biol. 74, 666 (2012).
[31] T. Glimm, J. Zhang, and Y.-Q. Shen, Stability of Turing-type patterns in a reaction-diffusion system with an external gradient, Int. J. Bifurcation Chaos 27, 1750003 (2017).

[32] Y. Mau, A. Hagberg, and E. Meron, Spatial Periodic Forcing Can Displace Patterns It Is Intended to Control, Phys. Rev. Lett. 109, 034102 (2012).

[33] K. Page, P. K. Maini, and N. A. M. Monk, Pattern formation in spatially heterogeneous Turing reaction-diffusion models, Physica D: Nonlinear Phenomena 181, 80 (2003).

[34] A. L. Krause, V. Klika, T. E. Woolley, and E. A. Gaffney, From one pattern into another: Analysis of Turing patterns in heterogeneous domains via WKBJ, arXiv:1908.7219.

[35] V. Klika, Significance of non-normality-induced patterns: Transient growth versus asymptotic stability, Chaos 27, 073120 (2017).

[36] V. Klika, M. Kozák, and E. A. Gaffney, Domain size driven instability: Self-organization in systems with advection, SIAM J. Appl. Math. 78, 2298 (2018).

[37] A. L. Krause, V. Klika, T. E. Woolley, and E. A. Gaffney, Heterogeneity induces spatiotemporal oscillations in reactiondiffusion systems, Phys. Rev. E 97, 052206 (2018). 